\title{
Faced Block
}

National Cancer Institute

\section{Source}

National Cancer Institute. Faced Block. NCI Thesaurus. Code C128636.

A block that has been cut down to the desired tissue plane, with the overlaying support

material removed. 